\title{
Asociación entre polifarmacia y prescripción inapropiada según la clase social en adultos mayores de Bucaramanga
}

\section{Association between polypharmacy and inappropriate prescription according to social class in older adults in Bucaramanga}

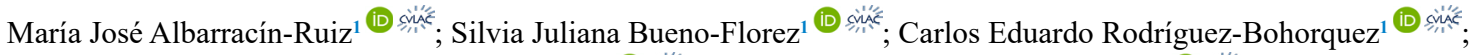

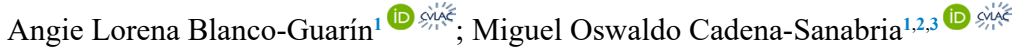

Forma de citar: Albarracín-Ruiz MJ; Bueno-Florez SJ; Rodríguez-Bohorquez CE; Blanco-Guarín AL; Cadena-Sanabria MO. Asociación entre Polifarmacia y Prescripción inapropiada según la clase social en adultos mayores de Bucaramanga. Salud UIS. 53: e21021. doi: https://doi.org/10.18273/saluduis.53.e:21021 @c) (i)

\section{Resumen}

Introducción: la polifarmacia y la prescripción inapropiada (PI) se han visto relacionadas con múltiples variables, incluidas las de aspecto socioeconómico, sin embargo, la evidencia en esta asociación es escasa y heterogénea. Objetivo: evaluar la asociación entre la polifarmacia y la prescripción inapropiada de medicamentos en adultos mayores de 60 años según su clase social. Material y métodos: Estudio piloto de diseño descriptivo, corte transversal realizada en la ciudad de Bucaramanga-Colombia, con una muestra de 135 adultos mayores de 60 años. Se realizó una encuesta donde se registraron datos sociodemográficos, estado de salud en el último mes, comorbilidades, medicamentos de uso crónico y escalas de funcionalidad. Resultados: no se encontró asociación estadísticamente significativa entre prescripción inapropiada y polifarmacia con la clase social y el nivel educativo $(\mathrm{P}=0,639)$. Sin embargo, el tener una filiación al sistema de seguridad social de tipo subsidiado estuvo significativamente asociada a la infra prescripción $(\mathrm{P}<0,029)$. La presencia de ingresos mensuales estuvo asociada con la presencia de polifarmacia $(\mathrm{P}<0,029)$ y prescripción inadecuada $(\mathrm{P}<0,033)$. Conclusiones: Existe una asociación entre algunas variables socioeconómicas, el número de médicos tratantes y algunas especialidades médicas con presentar polifarmacia y prescripción inapropiada de medicamentos.

Palabras clave: Polifarmacia; Anciano; Clase Social; Prescripción Inadecuada; Prevalencia; Impacto de Enfermedad; Factores Epidemiológicos; Errores de Medicación; Comorbilidad; Fragilidad.

1. Universidad Autónoma de Bucaramanga. Santander, Colombia.

2. Clínica FOSCAL, Bucaramanga. Colombia.

3. Universidad Industrial de Santander, Bucaramanga, Colombia.

Correspondencia: María José Albarracín Ruiz Dirección: Calle 147 22-117 Floridablanca, Santander casa 21. Teléfono: 3012379591 Correo electrónico:malbarracin617@unab.edu.co 


\begin{abstract}
Introduction: Polypharmacy and inappropriate prescribing have been related to multiple variables, including sociodemographic, however the evidence is limited and heterogeneous. Objective: To evaluate the association between polypharmacy and inappropriate prescribing in adults over 60 years old according to their social class. Material and methods: A pilot cross-sectional population-based study in the city of Bucaramanga, Colombia, with a sample of 135 adults over 60 years old. A survey was conducted where sociodemographics, health status in the last month, comorbidities, drugs of chronic use and functionality according to scales were registered. Results: No statistical association was found between inappropriate prescribing and Polypharmacy amongst social class and education level $(\mathrm{P}=0.639)$. Nevertheless, having an affiliation with a subsidized social security system was significantly associated with infra prescription $(\mathrm{P}<0.029)$. The existence of monthly income was associated with the presence of polypharmacy $(\mathrm{P}<0.029)$ and inadequate prescribing $(\mathrm{P}<0.033)$. Conclusions: There exists an association between some socioeconomic variables, the number of practicing physicians and some medical specialties with polypharmacy and inadequate prescription of drugs.
\end{abstract}

Keywords: Polypharmacy; Aged; Social Class; Inappropriate Prescribing; Prevalence; Sickness Impact; Epidemiologic Factors; Medication Errors; Comorbidity; Frailty.

\section{Introducción}

En Colombia durante el año 2013, el Ministerio de Salud reportó que el $10,53 \%$ de la población es mayor de 65 años, se estima que para el 2020 habría un crecimiento mayor al $3,76 \%$ en este grupo etario ${ }^{1}$. Además, resaltó que el $80 \%$ de los adultos mayores tienen al menos una enfermedad crónica, lo que aumenta la necesidad del uso de varios medicamentos ${ }^{2}$.

El consumo de 5 o más medicamentos se considera polifarmacia ${ }^{3,4}$, esto genera diferentes consecuencias en los adultos mayores, entre ellas la prescripción inapropiada de medicamentos (PI) definida como una práctica donde el riesgo de sufrir efectos adversos es superior al beneficio clínico. La PI se define como "el uso de fármacos con una mayor frecuencia o duración de la indicada, el uso de fármacos con un elevado riesgo de interacciones medicamento-medicamento o medicamento-enfermedad, $\mathrm{y}$ fármacos duplicados o de la misma clase y la no utilización de fármacos beneficiosos que sí están clínicamente indicados, pero que a menudo no se prescriben en pacientes mayores por diferentes razones, no siempre bien determinadas" 5 . Estos síndromes se han relacionado con variables socioeconómicas, entre ellas el nivel de ingresos o el tipo de vivienda.

Hay estudios que establecen relación entre polifarmacia y variables socioeconómicas. A nivel mundial se encontró una prevalencia de la polifarmacia del $9 \%$ y un predominio entre los adultos con bajo estrato socioeconómico, desempleados y edad más avanzada ${ }^{6}$. En un estudio realizado en Turquía se demostró que en población adulta institucionalizada existía una relación estadísticamente significativa entre el número de hijos y una mayor prevalencia de polifarmacia ${ }^{7}$.

A nivel de Latinoamérica un estudio en Chile reportó PI nivel 1 (medicamentos que no se deben usar) en un 9,7\% (IC95\%: 7,1\%-13,1\%) de acuerdo con los criterios de BEERS asociado con polifarmacia con un OR de 2,8 (IC95\%: 1,3-6,1). Sin embargo, el nivel educacional, la zona de vivienda urbano-rural, la edad y la presencia de médico de cabecera no resultaron ser predictores estadísticamente significativos del uso inapropiado ${ }^{8}$.

En Argentina, un estudio obtuvo una media de 6,1 medicamentos de uso regular, sin diferencias entre género, siendo mayor el número de medicamentos en adultos mayores de 80 años y de acuerdo a los criterios de BEERS mostró un $66 \%$ de prescripción inapropiada, sin asociación con el nivel socioeconómico?.

En Colombia, un estudio realizado en la ciudad de Bogotá relacionó la polifarmacia y las características sociodemográficas de la población de la muestra total, $548(27,4 \%)$ tenían polifarmacia y se vio más relacionada con el sexo femenino y adultos mayores ${ }^{10}$.

Adicionalmente, en otro estudio realizado en Colombia sobre PI en el adulto mayor en consulta externa de primer nivel, se encontró que $21,5 \%$ tenían uso de medicación potencialmente inapropiada de acuerdo con los criterios de BEERS, adicionalmente en esta población se encontró polifarmacia en $34,8 \%$ de los pacientes ${ }^{11}$. 
A pesar de que la evidencia en cuanto a polifarmacia y prescripción inapropiada de medicamentos tiene resultados heterogéneos, en la literatura mundial se ha documentado consecuencias negativas en la salud del adulto mayor como: reacciones adversas a medicamentos que ocasionan hasta un $10 \%$ de consultas a urgencias y hasta en un $38 \%$ amenazan la vida ${ }^{12}$, interacciones medicamentosas que se presentan en un 35 a $60 \%$ de los casos y aumentan exponencialmente con cada medicamento ${ }^{13}$, cascadas de prescripción como el uso de nuevos medicamentos para modular efectos adversos de otro, la no adherencia a los tratamientos médicos y el incremento de síndromes geriátricos con deterioro cognitivo, caídas, desnutrición y $\mathrm{PI}^{14}$.

Pese a que los resultados de los estudios más recientes son heterogéneos, existe evidencia que vincula una asociación entre la polifarmacia y la PI con variables socioeconómicas. Siendo Colombia un país en vía de desarrollo con alta desigualdad social, esto podría acentuar dicha asociación, por lo que en este artículo se busca determinar la asociación entre la polifarmacia y la prescripción inadecuada de medicamentos en adultos mayores de 60 años según su clase social.

\section{Metodología}

Se llevó a cabo un estudio piloto de diseño descriptivo y corte transversal que tuvo lugar en la ciudad de Bucaramanga y su área Metropolitana, durante el año 2017.

Se realizó un muestreo por conveniencia donde se seleccionaron al azar barrios pertenecientes a cada estrato socioeconómico. La captación se programó en jornadas conformadas por uno o más entrevistadores durante un periodo de 6 meses, donde la encuesta era aplicada en cada vivienda o en el caso de la existencia de un parque comunitario en este.

Se definió incluir en el estudio adultos mayores de 60 años residentes en la ciudad de Bucaramanga y Área Metropolitana durante el año 2017 que hayan dado su consentimiento para participar por medio del diligenciamiento del consentimiento informado escrito realizado este último de acuerdo con las consideraciones éticas y la respectiva ovación del comité de ética.

Se excluyeron del estudio adultos mayores hospitalizados, ingreso a urgencias en las últimas 48 horas, diagnóstico previo de demencia en estadio severo, institucionalización en hogares geriátricos, ausencia de familiares o cuidadores en el momento de la entrevista.
Para la aplicación de la encuesta los entrevistadores recibieron un entrenamiento, el cual consistió en la simulación de la encuesta donde se explicaba cada pregunta, la interpretación de los instrumentos de medición y se tomó nota del tiempo y la metodología empleados por los encuestadores.

Se utilizó una encuesta diseñada por los autores que incluía variables sociodemográficas como estado civil, edad, sexo, afiliación al sistema de salud, estrato socioeconómico caracterizado de 1 a 6 de acuerdo a la organización local, tipo de vivienda, nivel educativo, pertenencia a redes de apoyo, estado laboral, e ingresos determinado por intervalos entre otras; variables relacionadas al estado de salud en el último mes, necesidad de usar servicios de salud, comorbilidades existentes, medicamentos farmacológicos o herbales de uso crónico (con dosis y vías de administración).

Se aplicaron 5 escalas (validadas en Colombia) que evalúan diferentes aspectos como: funcionalidad del adulto mayor por medio del índice de Barthel ${ }^{15} \mathrm{y}$ escala de Lawton y Brody ${ }^{16}$; grado de comorbilidad por medio del índice de Charlson ${ }^{17}$; valoración del riesgo social por medio de la escala de Gijón ${ }^{18}$; tamizaje de deterioro cognitivo medio de mini-mental test ${ }^{19}$; identificación de infra prescripción, prescripción inapropiada 0 polifarmacia por medio de la aplicación de los Criterios STOPP/START $2014^{20}$.

Se definió como infraprescripción a la omisión de un manejo farmacológico indicado para una condición médica determinada, prescripción inapropiada al uso de fármacos con una frecuencia mayor o mayor duración de la indicada y polifarmacia al uso de 5 o más medicamentos.

Para el presente estudio se determinó el término clase social para abarcar el entorno socioeconómico y estratificación social.

Para el análisis estadístico se emplearon medidas de frecuencia, tendencia central como media o mediana, con su respectiva desviación estándar o rango interquartil según su distribución. Para evaluar diferencias estadísticas entre las proporciones se utilizó la prueba exacta de Fischer. Se consideró una asociación significativa si se encontraba un valor de $\mathrm{p}<0,05$.

Esta investigación se realizó bajo los conceptos éticos de la normativa colombiana. 


\section{Resultados}

El presente estudio contó con una muestra de 135 adultos mayores de 60 años, con una media de edad de 70,83 años, $31,85 \%$ tenían 80 o más años, $72,59 \%$ de la muestra eran mujeres, $27,40 \%$ eran hombres, $51,11 \%$ estaban afiliados al régimen subsidiado, $30,37 \%$ estaban afiliados al régimen contributivo, de acuerdo a la clase social prevalecieron los estratos 1 y 2 con $32,59 \%$ y $18,51 \%$ respectivamente, el $44,44 \%$ tenían estudios en básica primaria, el $54,07 \%$ tienen ingresos económicos mensuales activos con ingresos mensuales menor al salario mínimo mensual vigente en la mayoría de los casos. (Tabla 1)

Tabla 1. Características sociodemográficas de la población.

\begin{tabular}{|c|c|c|c|}
\hline Variable & Categoría & $n=135$ & $\%$ \\
\hline \multirow{6}{*}{ Edad } & 60 a 65 & 39 & $28,88 \%$ \\
\hline & 65 a 70 & 24 & $17,77 \%$ \\
\hline & 71 a 75 & 29 & $21,48 \%$ \\
\hline & 76 a 85 & 29 & $21,48 \%$ \\
\hline & 86 a 90 & 13 & $9,62 \%$ \\
\hline & $>91$ & 1 & $0,74 \%$ \\
\hline \multirow{2}{*}{ Género } & Femenino & 98 & $72,59 \%$ \\
\hline & Másculino & 37 & $27,40 \%$ \\
\hline \multirow{4}{*}{ Estado civil } & Casado & 58 & $42,96 \%$ \\
\hline & Soltero & 20 & $14,81 \%$ \\
\hline & Viudo & 40 & $29,62 \%$ \\
\hline & Divorciado & 17 & $12,59 \%$ \\
\hline \multirow{5}{*}{ Afiliación al SSS } & subsidiado & 69 & $51,11 \%$ \\
\hline & contributivo & 41 & $30,37 \%$ \\
\hline & especial/prepagada & 15 & $11,11 \%$ \\
\hline & vinculado/sisben & 5 & $3,70 \%$ \\
\hline & Ninguno & 5 & $3,70 \%$ \\
\hline \multirow{6}{*}{ Clase social } & 1 & 44 & $32,59 \%$ \\
\hline & 2 & 25 & $18,51 \%$ \\
\hline & 3 & 23 & $17,03 \%$ \\
\hline & 4 & 17 & $12,59 \%$ \\
\hline & 5 & 13 & $9,62 \%$ \\
\hline & 6 & 13 & $9,62 \%$ \\
\hline \multirow{6}{*}{ Nivel educativo } & ninguno & 20 & $14,81 \%$ \\
\hline & Primaria & 60 & $44,44 \%$ \\
\hline & Secundaria & 29 & $21,48 \%$ \\
\hline & técnica/tecnología & 8 & $5,92 \%$ \\
\hline & Pregrado & 9 & $6,66 \%$ \\
\hline & Postgrado & 9 & $6,66 \%$ \\
\hline \multirow{2}{*}{$\begin{array}{l}\text { Ingresos económicos } \\
\text { mensuales }\end{array}$} & $\mathrm{Si}$ & 73 & $54,07 \%$ \\
\hline & No & 62 & $45,92 \%$ \\
\hline \multirow{6}{*}{$\begin{array}{l}\text { Monto de los } \\
\text { ingresos }\end{array}$} & no aplica & 62 & $45,92 \%$ \\
\hline & menos de $\$ 644350$ & 23 & $17,03 \%$ \\
\hline & $\$ 644350$ a $\$ 1000000$ & 11 & $8,14 \%$ \\
\hline & $\$ 1000100$ a $\$ 3000000$ & 26 & $19,25 \%$ \\
\hline & $\$ 3000100$ a $\$ 5000000$ & 5 & $3,70 \%$ \\
\hline & $>\$ 5000000$ & 8 & $5,92 \%$ \\
\hline
\end{tabular}

En la Tabla 2 se observa la asociación entre la prescripción inapropiada, polifarmacia e infra prescripción de medicamentos a adultos mayores según la clase social y/o nivel socioeconómico al cual pertenecen, no fue estadísticamente significativa ( $\mathrm{P}=0,648 ; \mathrm{P}=0,782 ; \mathrm{P}=0,113$ respectivamente), el nivel educativo de los participantes tampoco fue un actor asociado significativo $(\mathrm{P}=0,639)$. Sin embargo, el tener una afiliación al sistema de seguridad social de tipo subsidiado estuvo significativamente asociada a la infra prescripción $(\mathrm{P}<0,029)$, así como se evidencio que los adultos mayores que tenían ingresos mensuales independientemente del monto de ingresos, con respecto a los que no contaban con ingresos propios se vio asociado con la presencia de polifarmacia $(\mathrm{P}<0,029)$ y prescripción inadecuada $(\mathrm{P}<0,033)$.

En la Tabla 3 se observa que se encontró que los medicamentos de uso más frecuente que se asociaron con la presencia de polifarmacia son los broncodilatadores en un $62,5 \%(\mathrm{P}=<0,01)$, seguido por los antidiabéticos en un $60 \%(\mathrm{P}=<0,01)$, cardioprotectores $55,17 \%$ $(\mathrm{P}=<0,01)$. En el caso de prescripción inapropiada, se encontró que el grupo de medicamentos más frecuentemente asociados fueron los analgésicos $53,85 \%(\mathrm{P}=<0,001)$ seguido por broncodilatadores $50 \%(\mathrm{P}=0,155)$, antidiabéticos $45 \%(\mathrm{P}=0,064)$.

Evaluamos la funcionalidad del adulto mayor por medio de escalas estandarizadas, encontrando que según el índice de Barthel, el 80\% (108) de los adultos mayores eran independientes, de los cuales un 58,82\% presentaba polifarmacia y $35,29 \%$ prescripción inapropiada, similares resultados se observan al aplicar la escala de Lawton y Brody, la cual encontró que la mayoría de adultos mayores eran independientes con 73,3\% (99). Sin embargo, al evaluar si existía una asociación significativa entre estas dos escalas, polifarmacia y prescripción inapropiada, el número de la muestra no fue representativo para hallar dicha asociación.

Adicionalmente, se encontró que el 71,85\% (97) no presentaba un deterioro cognitivo, de estos el 19,58\% tenía polifarmacia y $26,8 \%$ prescripción inapropiada, sin embargo, la asociación de estas variables tampoco pudo establecerse.

Igualmente, al evaluar el posible riesgo social medido por la escala de Gijón, la mayoría de adultos mayores presentaban un buen ambiente y apoyo social $(57,7 \%)$, sin embargo, la asociación entre el riesgo social con polifarmacia y prescripción inapropiada no fue significativa ( $\mathrm{P}=0.550$ y $\mathrm{P}=1.000$ respectivamente). 
Tabla 2. Asociación entre variables socioeconómicas, polifarmacia, prescripción inapropiada e infra prescripción.

\begin{tabular}{|c|c|c|c|c|c|c|c|}
\hline Clase social & $\mathbf{n}$ & $\%$ polifarmacia & $\mathbf{p}$ & $\begin{array}{c}\text { \% Prescripción } \\
\text { inapropiada }\end{array}$ & $\mathbf{p}$ & $\%$ infra prescripción & $\mathbf{p}$ \\
\hline 1 & 44 & 18,18 & & 27,27 & & 22,73 & \multirow{6}{*}{0,113} \\
\hline 2 & 25 & 28 & & 40 & & 28 & \\
\hline 3 & 23 & 21,74 & \multirow{4}{*}{0,782} & 17,39 & 0648 & 8,7 & \\
\hline 4 & 17 & 23,53 & & 23,53 & טוס, & 0 & \\
\hline 5 & 13 & 7,69 & & 30,77 & & 23 & \\
\hline 6 & 13 & 23,08 & & 30,77 & & 7,69 & \\
\hline \multicolumn{8}{|l|}{ Nivel educativo } \\
\hline Ninguno & 20 & 25 & \multirow{6}{*}{0,639} & 25 & & 25 & \multirow{6}{*}{0,12} \\
\hline Primaria & 60 & 25 & & 28,33 & & 23,33 & \\
\hline Secundaria & 29 & 10,34 & & 31.03 & 0,972 & 6.9 & \\
\hline Técnica/Tecnología & 8 & 25 & & 37,5 & & 25 & \\
\hline Pregrado & 9 & 11,11 & & 22,22 & & 0 & \\
\hline Postgrado & 9 & 22,22 & & 22,22 & & 0 & \\
\hline \multicolumn{8}{|l|}{$\begin{array}{l}\text { Afiliación a régimen } \\
\text { de seguridad social }\end{array}$} \\
\hline Contributivo & 69 & 23,19 & & 36,23 & & 8,7 & \\
\hline Subsidiado & 41 & 24,39 & \multirow{4}{*}{0,448} & 24,39 & & 31,71 & \multirow{4}{*}{0,029} \\
\hline Especial/prepagada & 15 & 6,67 & & 20 & 0,14 & 20 & \\
\hline Vinculado/sisben & 5 & 20 & & 0 & & 20 & \\
\hline Ninguno & 5 & 0 & & 0 & & 0 & \\
\hline \multicolumn{8}{|l|}{ Ingresos mensuales } \\
\hline $\mathrm{Si}$ & 73 & 13,7 & \multirow{2}{*}{0,029} & 20,55 & \multirow{2}{*}{0,033} & 13,7 & \multirow{2}{*}{0,263} \\
\hline No & 62 & 29,03 & & 3.1 & & 20,97 & \\
\hline \multicolumn{8}{|l|}{$\begin{array}{l}\text { Monto de ingresos } \\
\text { mensuales }\end{array}$} \\
\hline Ninguno & 62 & 29,03 & \multirow{6}{*}{0,095} & 37,1 & \multirow{6}{*}{0,253} & 20,97 & \multirow{6}{*}{0,261} \\
\hline Menos de 644350 & 23 & 17,39 & & 13,04 & & 26,09 & \\
\hline 644351 a 1000000 & 11 & 9,09 & & 27,27 & & 0 & \\
\hline 1000100 a 3000000 & 26 & 3,85 & & 19,23 & & 11,54 & \\
\hline 3000100 a 5000000 & 5 & 40 & & 20 & & 20 & \\
\hline Más de 5000100 & 8 & 25 & & 37,5 & & 0 & \\
\hline
\end{tabular}

Tabla 3. Asociación entre medicamentos de uso diario, polifarmacia y prescripción inapropiada.

\begin{tabular}{lcccc}
\hline \multicolumn{1}{c}{ Grupo de medicamentos } & Polifarmacia n (\%) & P & Prescripción inapropiada n (\%) & P \\
\hline Antidiabéticos & $12(60)$ & $<0,001$ & $9(45)$ & 0,064 \\
Antihipertensivos & $24(37,5)$ & $<0,001$ & $20(31,25)$ & 0,284 \\
Analgésico no esteroides & $9(34,62)$ & 0,054 & $14(53,85)$ & 0,001 \\
Medicamentos usados en desordenes tiroides & $9(37,5)$ & 0,03 & $5(20,83)$ & 0,27 \\
Antiagregantes & $14(46,67)$ & $<0,001$ & $11(36,67)$ & 0,171 \\
Protectores gastricos & $7(38,89)$ & 0,048 & $5(6,78)$ & 0,607 \\
Broncodilatadores & $5(62,5)$ & 0,01 & $4(50)$ & 0,155 \\
Cardioprotectores & $16(55,17)$ & $<0,001$ & $9(31,03)$ & 0,43 \\
Otros & $18(34,62)$ & 0,002 & $26(50)$ & $<0.001$ \\
\hline
\end{tabular}


Finalmente, el $85(62,96 \%)$ de los adultos mayores presentan un índice de 0 puntos según el índice de Charlson para una mortalidad de $12 \%$ a un año, nuevamente no pudiéndose establecer asociación estadística según la muestra obtenida.

Para evaluar la asociación entre las distintas patologías prevalentes en adultos mayores con polifarmacia y prescripción inapropiada, se realizó una clasificación de las enfermedades para agruparlas de acuerdo con el órgano o sistema afectado, y se ubicó en la categoría otras enfermedades aquellas que no cumplían con las características de las clasificaciones establecidas. En la Tabla 4 se encontró que las enfermedades respiratorias tuvieron la mayor frecuencia de polifarmacia con un $71,43 \%$ de la población encuestada siendo una asociación significativa $(\mathrm{P}=<0,01)$, seguido por las enfermedades psiquiátricas y neurológicas con un $50 \%$ cada una, respectivamente $(\mathrm{P}=0,19 \mathrm{P}=0,67)$. El caso de la asociación de prescripción inapropiada y las distintas enfermedades fue más frecuente en las enfermedades psiquiátricas que se encontró en un $75 \%(\mathrm{P}=0,06)$, seguido por las enfermedades respiratorias con un $42,86 \%(\mathrm{P}=0,40)$, es importante mencionar que ninguna enfermedad se asoció de forma estadísticamente significativa a la prescripción inapropiada.

Tabla 4. Asociación entre enfermedades prevalentes en el adulto mayor, polifarmacia y prescripción inapropiada.

\begin{tabular}{lcccc}
\hline Enfermedad & $\begin{array}{c}\text { Polifarmacia } \\
\mathbf{n}(\%)\end{array}$ & $\mathbf{P}$ & $\begin{array}{c}\text { Prescripción } \\
\text { inapropiada } \\
\mathbf{n}(\%)\end{array}$ & $\mathbf{P}$ \\
\hline Endometabólicas & $19(38,78 \%)$ & $<0,01$ & $16(32,65 \%)$ & 0,42 \\
Osteomusculares & $10(30,30 \%)$ & 0,14 & $12(36,36 \%)$ & 0,26 \\
Psiquiátricas & $2(50 \%)$ & 0,19 & $3(75 \%)$ & 0,06 \\
Respiratorias & $5(71,43 \%)$ & $<0,01$ & $3(42,86 \%)$ & 0,40 \\
Cardiovasculares & $25(36,23 \%)$ & $<0,01$ & $24(34,78 \%)$ & 0,08 \\
Gastrointestinales & $4(21,05 \%)$ & 1,00 & $7(36,84 \%)$ & 0,41 \\
Neurológicas & $3(50 \%)$ & 0,10 & $2(33,33 \%)$ & 0,67 \\
Renales & $1(25 \%)$ & 1,00 & $1(25 \%)$ & 1,00 \\
Otras & $16(27,59 \%)$ & 0,13 & $14(24,14 \%)$ & 0,44 \\
\hline
\end{tabular}

En la Tabla 5 se describe la relación entre prescripción inapropiada y polifarmacia, la cual se encontró estadísticamente significativa $(\mathrm{P}=0,004)$. Adicionalmente, se evaluó la asociación entre el número de médicos tratantes y la prescripción inapropiada, bajo la idea de que múltiples comorbilidades en un mismo paciente necesitan visitar a más de un médico tratante, siendo esta no significativa $(\mathrm{P}<0,519)$.
Tabla 5. Asociación entre Polifarmacia, número de médicos tratantes y prescripción inapropiada.

\begin{tabular}{lcc}
\cline { 2 - 3 } & $\begin{array}{c}\text { Prescripción } \\
\text { inapropiada }\end{array}$ & P \\
\hline Polifarmacia & 14 & 0,004 \\
Número de médicos tratantes & 6 & 0,519 \\
\hline
\end{tabular}

De igual forma, se realizó un análisis sobre la asociación entre polifarmacia y prescripción inapropiada y el tipo de especialidad, los resultados se encuentran en la Tabla 6, donde encontró que las especialidades cardiología, nefrología, medicina interna, neumología, ortopedia y nutrición se asociaron significativamente a la presencia de polifarmacia con una $\mathrm{p}<0,05$, sin embargo para el caso de prescripción inapropiada solamente la especialidad de cardiología y ortopedia se asociaron de forma significativa. Además, se encontró que, en ninguno de los encuestados, las especialidades como reumatología, oncología, fisiatría, endocrinología, presentaban casos de polifarmacia o prescripción inapropiada por lo cual no se incluyeron en la tabla.

Tabla 6. Asociación entre polifarmacia, prescripción inapropiada y especialidad médica.

\begin{tabular}{lcccc}
\hline \multicolumn{1}{c}{ Especialidad } & $\begin{array}{c}\text { Polifarmacia } \\
\text { n (\%) }\end{array}$ & $\mathbf{P}$ & $\begin{array}{c}\text { Prescripción } \\
\text { inapropiada } \\
\mathbf{n}(\%)\end{array}$ & $\mathbf{P}$ \\
\hline Cardiología & $5(17,86)$ & 0,035 & $6(15,79)$ & 0,042 \\
Gastroenterología & $1(3,57)$ & 0,586 & $1(2,63)$ & 0,84 \\
Nefrología & $1(3,57)$ & 0,05 & $1(2,63)$ & 109 \\
Urología & $1(3,57)$ & 0,586 & $1(2,63)$ & 0,84 \\
Medicina interna & $10(35,71)$ & $<0,01$ & $7(18,42)$ & 0,903 \\
Neumología & $4(14,29)$ & 0,015 & $3(7,89)$ & 0,374 \\
Ortopedia & $4(14,29)$ & 0,005 & $4(10,53)$ & 0,032 \\
Nutrición & $1(3,57)$ & 0,05 & $1(2,63)$ & 0,109 \\
Psiquiatría & $1(3,57)$ & 0,586 & $2(5,26)$ & 0,134 \\
Oftalmología & $4(14,29)$ & 0,868 & $4(10,53)$ & 0,548 \\
Neurología & $0(0,00)$ & 0,299 & $0(0,00)$ & 0,204 \\
Otros & $7(25)$ & 0,041 & $5(13,16)$ & 0,97 \\
\hline
\end{tabular}

\section{Discusión}

La polifarmacia y la prescripción inapropiada hacen parte de los síndromes geriátricos, los cuales se han visto relacionados con múltiples variables, entre estas las de tipo socioeconómicas. Sus desenlaces desfavorables a la salud de los pacientes adultos mayores han sido ampliamente estudiados, sin embargo, la evidencia es escasa y heterogénea. 
Este estudio buscó establecer la asociación entre la polifarmacia y la prescripción inadecuada de medicamentos en adultos mayores de 60 años según su clase social, así como otras variables socioeconómicas descritas anteriormente.

Se encontró que contar con ingresos económicos mensuales, independientemente del monto de estos, vs los que no tenían ingresos propios, está significativamente asociado con polifarmacia $(\mathrm{P}=0,029)$ y prescripción inadecuada $(\mathrm{P}=0,033)$. De igual forma, se encontró que el pertenecer a un régimen de salud subsidiado se asociaba a la presencia de infra prescripción ( $\mathrm{P}=0,263)$. En relación con lo anterior, se observaron resultados similares en un estudio realizado en China donde se comparó una población con bajo acceso al servicio de salud de atención primaria, que presentó mayor polifarmacia, comparada con la que sí tuvo acceso a la misma ${ }^{21}$.

En el análisis realizado se evaluó la asociación entre la variable nivel educativo y las 3 variables principales (polifarmacia, PI, infra prescripción). Se encontró que, pese a que esta relación no fue significativa, es de resaltar que la mayoría de adultos mayores encuestados tenían un nivel educativo bajo. Un estudio realizado en Suiza con una muestra de 621 adultos mayores de 77 años obtuvo como resultado un $42,4 \%$ de adultos con polifarmacia, con una media de 4,4 medicamentos de uso por persona, la cual aumentaba a 4,6 en los pacientes con menor educación. Sin embargo, al ajustar según edad, sexo, comorbilidades y condición de vivienda no hubo relación; así mismo, no se encontró asociación entre polifarmacia y ocupación o ingresos ${ }^{22}$. De igual forma en Chile, con una muestra de 1048 adultos mayores, se estimó la relación de factores sociodemográficos y uso inapropiado de medicamentos, encontrándose que el nivel educativo no resultó ser un predictor estadísticamente significativos del uso inapropiado ${ }^{8}$.

Dentro de los resultados del presente estudio se encontró que la presencia de ciertas comorbilidades se asociaba a la coexistencia de polifarmacia y prescripción inapropiada en el adulto mayor, como lo es el caso de las enfermedades respiratorias, que fueron las más repetidas con una frecuencia de $71,42 \%$ siendo estadísticamente significativa $(\mathrm{P}=<0,01)$; para el caso de la prescripción inapropiada, fue más frecuentemente encontrada en aquellas personas con enfermedades psiquiátricas $75 \%(\mathrm{P}=0,06)$, sin embargo, no fue una asociación significativa. Kutsal, et al. en un estudio multicéntrico de 1430 adultos mayores en Turquía encontró que la polifarmacia se correlaciona con la presencia de enfermedades crónicas, donde la distribución del número de medicamentos variaba significativamente con la presencia de estas condiciones médicas $(\mathrm{P}<0,001)^{7}$. De igual forma, Corsello, en el estudio GIFA 1 en Italia, estudió la relación entre la medicación previa al ingreso hospitalario, medicación al alta hospitalaria y la presencia de comorbilidades, sus resultados arrojaron que tener más de 4 diagnósticos médicos o alguna patología crónica se relaciona de forma significativa $(\mathrm{P}<0,005)$ con polifarmacia al alta hospitalaria ${ }^{23}$. Vyas A. también encontró que la polifarmacia era directamente proporcional con el número de comorbilidades y por ende se presentaba un menor riesgo de polifarmacia en pacientes con 1 o 2 de ellas con un OR 0,04; es de resaltar la comparación por grupos, donde se encontró que la coexistencia de enfermedades cardiometabólicas y músculo esqueléticas en un paciente genera más riesgo de reportar polifarmacia ${ }^{24}$.

También se observó la relación entre el tipo de especialidad a las que acudían los adultos mayores y la polifarmacia, encontrándose una relación estadísticamente significativa con ambas variables. Estos resultados son similares a los encontrados en un estudio realizado en Argentina, en el cual los pacientes que veían 4 o más médicos se asociaban con el uso de 8 medicamentos en promedio ${ }^{25}$.

Por otra parte, se evaluó la asociación de grupos de medicamentos con polifarmacia y prescripción inapropiada, donde se encontró una asociación estadísticamente significativa $(\mathrm{P}<0,05)$ para ambas variables con fármacos como acetaminofén, anticonvulsivantes de primera generación, metilxantinas y antagonistas de los receptores de angiotensina II, éstos son medicamentos de alto uso en atención primaria para las patologías más frecuentemente encontradas en los adultos mayores. Se observan similitudes con estudios realizados en otros países con muestras representativas, donde, entre los fármacos más frecuentemente utilizados se resaltan complejos vitamínicos, ansiolíticos o psicóticos, analgésicos complejos vitamínicos, analgésicos, psicolépticos $\mathrm{y}$ anti hipertensivos, relacionando esta frecuencia con medicación potencialmente inapropiada ${ }^{26-28}$.

\section{Limitaciones del estudio}

Nuestro estudio tiene algunas limitaciones. En primera instancia, el tamaño de muestra fue pequeña y se vio limitada al tiempo y método de selección por conveniencia. Esto limitó la asociación estadística entre algunas variables, posibilitó la existencia de un sesgo de selección. 
Por otro lado, al momento de encuestar a los adultos mayores, algunos de ellos no contaban con historia clínica o fórmula médica, no se contó con una lista estandarizada de patologías ni de medicamentos a interrogar en cada entrevista, lo cual podría llevar un posible sesgo de información. Sin embargo, este estudio constituye uno de los pocos realizados en nuestro país donde se hayan incluido múltiples variables que hacen parte de las diferentes esferas del individuo, y que pueden influenciar en la presencia de polifarmacia o prescripción inapropiada.

Con base en nuestra experiencia, se recomienda la realización de estudios futuros donde se realice un cálculo de muestra representativa de la población de adulto mayor del área metropolitana de Bucaramanga, con el objetivo de reducir posibles sesgos metodológicos. Se deberá estandarizar un instrumento de recolección que permita, por un lado la verificación de datos en base a un documento legal como lo es la historia clínica, y la evaluación de la adherencia al manejo de medicamentos del adulto mayor, de tal forma que se pueda establecer la validez y realidad de los datos recolectados.

\section{Conclusiones}

El presente estudió permitió comprobar la asociación de algunas variables socioeconómicas como ingresos y afiliación al régimen subsidiado con prescripción inapropiada, polifarmacia e infra prescripción.

Por otro lado, se pudo determinar cómo las especialidades médicas y las comorbilidades más frecuentemente encontradas, como las enfermedades respiratorias y cardiovasculares y los fármacos de uso más frecuente, están directamente relacionadas con la prescripción inapropiada de medicamentos.

\section{Conflicto de interés}

Los autores no declaran ningún conflicto de interés.

\section{Referencias}

1. Ministerio de Salud y Protección Social. Envejecimiento Demográfico. Colombia 19512020. Dinámica Demográfica Y Estructuras Poblacionales. 2013; 48.

2. Higuera, C. A., Elsharkawy, K., Klika, A. K., Brocone, M., \& Barsoum WK, Prevention D, Promotion H, Health P. The State of Aging and Health in America 2013 is the sixth volume of a series that presents a snapshot of the health and aging landscape in the United States or another region of the world. This series presents the most current information and statistics. Clin Orthop Relat Res. 2013; 469(5): 1391-1400.

3. Gnjidic D, Hilmer SN, Blyth FM, Naganathan V, Waite L, Seibel MJ, et al. Polypharmacy cutoff and outcomes: Five or more medicines were used to identify community-dwelling older men at risk of different adverse outcomes. J Clin Epidemiol. 2012; 65(9): 989-995. doi: http://dx.doi.org/10.1016/j. jclinepi.2012.02.018

4. Onder G, Petrovic M, Tangiisuran B, Meinardi MC, Markito-Notenboom WP, Somers A, et al. Development and validation of a score to assess risk of adverse drug reactions among in-hospital patients 65 years or older. Arch Intern Med. 2010; 170(13): 1142-1148. doi: 10.1001/archinternmed.2010.153

5. Gurwitz JH. Neither Too Much nor Too Little. 1999; 281(2): 113-115.

6. Thomas HF, Sweetnam PM, Janchawee B, Luscombe DK. Polypharmacy among older men in South Wales. Eur J Clin Pharmacol. 1999; 55(5): 411-415.

7. Gokce Kutsal Y, Barak A, Atalay A, Baydar T, Kucukoglu S, Tuncer T, et al. Polypharmacy in the elderly: A multicenter study. J Am Med Dir Assoc. 2009; 10(7): 486-490.

8. Passi A, Margozzini P, Valenzuela E, Hoyl T, Marín PP, Carrasco M, et al. Uso inapropiado de medicamentos en adultos mayores: resultados de la Encuesta Nacional de Salud 2010. Rev Med Chil. 2016; 144(4): 417-425.

9. Marzi MM, Diruscio V, Nunez HM, Pires SM, Quaglia BN. Analysis of medication prescription in an Argentinian geriatric hospital. Rev Med Chil. 2013; 141(2): 194-201.

10. Cano-Gutierrez C, Samper-Ternent R, Cabrera J, Rosselli D. Medication use among older adults in Bogota, Colombia. Rev Peru Med Exp Salud Publica. 2016; 33(3): 419-424.

11. Holguín-Hernández E, Orozco-Díaz JG. Medicación potencialmente inapropiada en ancianos en un hospital de primer nivel, Bogotá 2007. Rev Salud Pública. 2010; 12(2): 287-299.

12. Gurwitz JH, Field TS, Harrold LR, Rothschild $\mathrm{J}$, Debellis K, Seger AC, et al. Incidence and preventability of in the ambulatory setting. J Am Med Assoc. 2003; 289(9): 1107-1116.

13. Shah BM, Hajjar ER. Polypharmacy, adverse drug reactions, and geriatric syndromes. Clin Geriatr Med. 2012; 28(2): 173-186. doi: http://dx.doi. org/10.1016/j.cger.2012.01.002 
14. Castro-Rodríguez J, Orozco-Hernández JP M-MD. Polifarmacia y prescripción de medicamentos potencialmente no apropiados en ancianos. Rev Méd Risaralda. 2016; 22(1): 52-57.

15. Solís C, Arrioja S, Manzano A. Índice de Barthel (IB): Un instrumento esencial para la evaluación funcional y la rehabilitación. 2005; 4: 1-6.

16. Lawton MP, Brody EM. Assessment of older people: self-maintaining and instrumental activities of daily living. Gerontologist. 1969; 9(3): 179-186. doi: https://doi.org/10.1093/geront/9.3_Part_1.179

17. Charlson ME, Pompei P, Ales KL, MacKenzie R. A new method of classifying prognostic in longitudinal studies: development and validation. J Chronic Diseases. 1987; 40: 373-383.

18. Cabrera D, Menenedez A, Fernandez A, Acebal V, Garcia J. Evaluación de la fiabilidad y validez de una escala de valoración social en el anciano Evaluation of the reliability and validity of a scale of social evaluation of the elderly. 1996; 1-9.

19. Rosselli D, Ardila A, Pradilla G, Morillo L, Bautista L, Rey O, et al. The Mini-mental state examination as a selected diagnostic test for dementia: a Colombian population study. GENECO. Rev Neurol. 1999; 30(5): 428-432.

20. Delgado Silveira E, Muñoz García M, Montero Errasquin B, Sánchez Castellano C, Gallagher PF, Cruz-Jentoft AJ. Prescripción inapropiada de medicamentos en los pacientes mayores: los criterios STOPP/START. Rev Esp Geriatr Gerontol. 2009; 44(5): 273-279.

21. Woo J, Zheng Z, Leung J, Chan P. Prevalence of frailty and contributory factors in three Chinese populations with different socioeconomic and healthcare characteristics. BMC Geriatr. 2015; 15(1): 1-11. doi: http://dx.doi.org/10.1186/s12877015-0160-7

22. Haider SI, Johnell K, Thorslund M, Fastbom J. Analysis of the association between polypharmacy and socioeconomic position among elderly aged $\geq 77$ years in Sweden. Clin Ther. 2008; 30(2): 419-427.

23. Corsonello A, Pedone C, Corica F, Incalzi RA. Polypharmacy in elderly patients at discharge from the acute care hospital. Ther Clin Risk Manag. 2007; 3(1): 197-203.

24. Vyas A, Pan X, Sambamoorthi U. Chronic condition clusters and polypharmacy among adults. Int J Family Med. 2012; 2012: 1-8.

25. Ascar GI, Hespe CB, Hernández MM. Relación entre polifarmacia y número de médicos consultados por pacientes ancianos. Rev Cuba Farm. 2015; 49(3): 491-501.
26. Mosegui GBG, Rozenfeld S, Veras RP, Vianna CMM. Avaliação da qualidade do uso de medicamentos em idosos. Rev Saude Publica. 1999; 33(5): 437-444.

27. Arey LB, Tremaine MJ, Monzingo FL. The numerical and topographical relations of taste buds to human circumv allate papillae throughout the life span. Anat Rec. 1935; 64(1): 9-25.

28. Lutz BH, Miranda VIA, Bertoldi AD. Potentially inappropriate medications among older adults in Pelotas, Southern Brazil. Rev Saude Publica. 2017; 51(0): 1-12. 